\title{
Low-Grade Endometrial Stromal
}

Sarcoma

Low-grade endometrial stromal sarcoma is a uterine sarcoma arising from the endometrial stroma.

For details $\rightarrow$ see Chap. 82 . 